\title{
SOME HYDRODYNAMICAL METHODS ${ }^{1}$
}

\author{
L. M. MILNE-THOMSON
}

The famous lines

Geography is about maps,

Biography is about chaps

leave little to be desired in conciseness. About what is mathematics? To use a hydrodynamical metaphor I shall take the plunge and assert that mathematics is about the logical consequences of assumed propositions, nowadays called axioms. Thus all mathematics is one. The fancied distinction between "pure" and "applied" is a modern and false dichotomy unknown to Euler and Cauchy. To see this clearly, reflect on the investigations to which the objects of nature have given rise. Insofar as they are mathematical, these investigations have been concerned, not with the objects of nature themselves, but with models, that is to say with certain ideal objects which are defined solely by a set of precise statements concerning the properties to be attributed to these objects. With such a set of statements the mathematician is on firm and familiar ground, and he can follow the path wherever it leads; perhaps to paradox or perhaps to such results as may induce the opinion, it can be no more, that his model furnishes an explanation of the physical counterpart which inspired the original postulates. From this point of view the ocean wave of the mathematician smacks as little of salt as does the Riemann Hypothesis. Indeed the flavor of fluid is a property from which no mathematical inference can be made, and so flavor, perhaps regrettably, must be consigned to the consideration of the chemist or the connoisseur.

1. Tensors. It is becoming increasingly realized that the most insight giving statement of the equations of motion of continuous media in general and of fluids in particular is by means of tensors.

There are at least two ways of regarding tensors, namely as quantities attached to a coordinate system, or as intrinsic entities, the latter way presenting many advantages not the least of which is the absence of an irrelevant coordinate system. Our intrinsic definition of a tensor of rank $n$ is recursive.

An address delivered before the Lexington meeting of the Society, November 30, 1956, by invitation of the Committee to Select Hour Speakers for Southeastern Sectional Meetings; received by the editors November 29, 1956.

1 The results presented in this paper were obtained in the course of research sponsored by the Office of Naval Research, Contract Nonr-567 [07]. 
Definition. A tensor or rank $n$ is a linear vector operator which, operating on an arbitrary vector $\mathbf{x}$ by scalar multiplication, gives rise to a tensor of rank $n-1$.

This definition together with the statement that a tensor of rank zero is a scalar, completely characterizes tensors of all positive integral rank.

Thus, for example, a vector $\boldsymbol{a}$ is a tensor of rank 1 since $\boldsymbol{a} \cdot \mathbf{x}$ is a scalar, or tensor of rank zero.

Similarly the dyadic product $\rho \boldsymbol{q} ; \boldsymbol{q}$ combines with $\mathrm{x}$ to give $\boldsymbol{\rho q}(\boldsymbol{q} \cdot \mathbf{x})$ a tensor of rank 1 and so $\rho q ; q$ is a tensor of rank 2, a 2-tensor, indeed the important momentum transfer tensor. The equation of steady motion of a fluid under no body forces can be written [1]

where

$$
\nabla \cdot[\Phi-\rho q ; q]=0,
$$

$$
\Phi=-p I-\frac{2}{3} \mu(\nabla \cdot q) I+\mu(\nabla ; q+q ; \nabla)
$$

is the stress tensor, $I$ being the idemfactor or unit 2-tensor.

From this form of the equation of motion, by integration over a sphere of large radius an expression for the force on a moving solid, for example an aerofoil, is readily obtained. The application of Oseen's approximation at a distance permits analysis of this force into a lift and drag. The drag is of particular interest since its expression [2] is $V F$ where $V$ is the velocity of the body and $F$ is an influx of liquid into the sphere, predominately an influx into the wake behind the body. Moreover the result is of an asymptotic character improving in accuracy as the radius of the sphere is increased. The two-dimensional form of this theorem was obtained by Filon [3] thirty years ago.

Tensor expression also pinpoints the Lagrangian form of the equation of motion [4]

$$
\frac{\partial ; \boldsymbol{r}}{\partial r_{0}} \cdot\left(\frac{\partial^{2} \boldsymbol{r}}{\partial t^{2}}-F\right)+\frac{1}{\rho} \cdot \frac{\partial p}{\partial x_{0}}=0,
$$

where $\boldsymbol{t}$ is the position vector at time $t$ of the particle originally at $\boldsymbol{r}_{0}$. The equation of continuity is

$$
\rho\left(\frac{\partial ; \boldsymbol{r}}{\partial r_{0}}\right)_{\mathrm{III}}=\rho_{0}
$$

where the notation indicates the third scalar invariant of the tensor derivative. 
Integration from 0 to $t$ leads directly to Weber's transformation

$$
\frac{\partial ; \boldsymbol{r}}{\partial r_{0}} \cdot q-q_{0}=-\frac{\partial \chi}{\partial r_{0}}
$$

where

$$
\chi=\int_{0}^{t}\left\{\int \frac{d p}{\rho}+\Omega-\frac{1}{2} q^{2}\right\} d t, \quad F=-\nabla \Omega .
$$

The Lagrangian form of the equation of motion has been applied to the one-dimensional motion of a gas, to two-dimensional steady motion and to free surface problems which I shall mention later.

The point that I want to make here is that the Lagrangian form is not quite so repulsive as the three equations which result from its expression in coordinates would seem to indicate. In the case of incompressibility we have $\rho=\rho_{0}$ and a consequent simplification. The equation should repay further study.

2. The theorems of Gauss and Stokes. The two great weapons of general fluid mechanics are the theorems of Gauss and Stokes [5] and their vector forms are suggestive;

$$
\int_{\tau} \nabla \circ X d \tau=\int_{S} d \mathbf{S} \circ X, \quad \int_{S}(d S \wedge \nabla) \circ X=\int_{C} d C \circ X,
$$

where, in the first the closed surface $S$ encloses the region $\tau$, and in the second the diaphragm $S$ spans the closed curve $C$. Here the small circle indicates scalar, vector, or dyadic multiplication and $X$ is a general function of position, scalar, vector, or tensor.

For the rate of change of circulation in a circuit which always consists of the same fluid particles we have

$$
\frac{d}{d t} \operatorname{circ} C=-\int_{C} d C \cdot \frac{1}{\rho} \nabla p=\int_{S} d S \cdot P,
$$

where $P=\nabla p \wedge \nabla(1 / \rho)$, so that $P$ is a vector along the intersection of surfaces of constant pressure and constant density. Also $\nabla P=0$, and so by Gauss's theorem $P$ defines tubes of constant intensity. Thus we have the famous meteorological theorem of Bjerknes [6] that the rate of change of circulation in $C$ is measured by the number of unit $\boldsymbol{P}$ tubes which $C$ embraces. When $p$ is a function of $\rho$ alone, $P=0$ and we obtain Kelvin's theorem on the constancy of circulation.

3. Complex variable. For plane flow the vector notation leads directly to the complex variable [7]. The use of the complex variable in 
two-dimensional problems has a long history, but it is only in recent years that full advantage has begun to be taken of the methods of function theory as opposed to resolution into equations in $x$ and $y$. What is beginning now to be more fully realized is that the variables most generally useful are not $x, y$ but the conjugate pair $z, \bar{z}$. For example if $\phi(x, y)$ is a plane harmonic function, it is the real part of a holomorphic function $f(z)$. The identity $2 \phi(x, y) \equiv f(z)+\bar{f}(\bar{z})$ leads, on putting $\bar{z}=0$, to

$$
f(z)=2 \phi\left(\frac{1}{2} z,-\frac{1}{2} i z\right)-\phi(0,0)+i \gamma,
$$

where $\gamma$ is an arbitrary real constant.

Again the circle theorem [8] states that the motion of an unbounded liquid whose complex potential is $f(z)$ when disturbed by the circle $|z|=a$ is governed by the complex potential

$$
w(z)=f(z)+\bar{f}\left(a^{2} / z\right),
$$

for on the boundary $\bar{z}=a^{2} / z$ so that the boundary is a streamline. That no new singularities are introduced is clear from the fact that of the points $z$ and $a^{2} / z$ only one lies inside the circular boundary and therefore if $f(z)$ has no singularities inside $|z|=a, \vec{f}\left(a^{2} / z\right)$ will have none outside.

The circle theorem enables us to deal with the perturbation produced by a cylinder of any form of cross-section, which can be mapped conformally on the circle.

From another point of view if the cross-section is the curve $\xi=\alpha$ in the net $z=n(\zeta), \zeta=\xi+i \eta$, we have $\zeta+\xi=2 \alpha$ on the boundary of the cross-section. Since $f(z)=f[n(\zeta)]=F(\zeta)$, the complex potential

$$
F(\zeta)+\bar{F}(2 \alpha-\zeta)
$$

makes $\psi=0$ on the boundary, but the second term may introduce new singularities. For example if the stream $U z$ is disturbed by the elliptic cylinder $\xi=\alpha$ in the net $z=c \cosh \zeta$, we shall have

$$
F(\zeta)+\bar{F}(2 \alpha-\zeta)=\frac{1}{2} U c\left\{e^{\zeta}+e^{-\zeta}+e^{2 \alpha-\zeta}+e^{-2 \alpha+\zeta}\right\} .
$$

The last term in the brackets introduces a singularity at infinity but the second term and the last are conjugate complex on the boundary and can therefore be omitted. Thus we get

$$
w=\frac{1}{2} U c\left(e^{\zeta}+e^{2 \alpha-\zeta}\right)=U c e^{\alpha} \cosh (\zeta-\alpha) .
$$


Similarly if the parabola $\xi=\alpha$ in the net $z=\zeta^{2}$ disturbs the stream $U z$, we have

$$
F(\zeta)+\bar{F}(2 \alpha-\zeta)=U \zeta^{2}+U(2 \alpha-\zeta)^{2}
$$

which gives at infinity the velocity $2 U$ instead of $U$. Halving gives

$$
w=\frac{1}{2} U \zeta^{2}+\frac{1}{2} U(2 \alpha-\zeta)^{2} .
$$

In the same line of thought we know that a stream function, say $\psi(z, \bar{z})$, can be defined for any two-dimensional flow whether irrotational or rotational, and the complex velocity is given by

$$
u-i v=-2 i \frac{\partial \psi}{\partial z} \text {. }
$$

In the case of steady streaming past a fixed cylinder, $\psi$ is constant on the boundary, and so

$$
\frac{\partial \psi}{\partial z} d z+\frac{\partial \psi}{\partial \bar{z}} d \bar{z}=0
$$

The Blasius theorem for the force $(X, Y)$ then gives [9]

$$
X-i Y=\frac{1}{2} i \rho \int_{c}(u-i v)(u+i v) d \bar{z}=-2 i \rho \int_{c}\left(\frac{\partial \psi}{\partial z}\right)^{2} d z,
$$

where the integral is taken round the contour $C$ of the cross-section. But the equation of $C$ is of the form $h(z, \bar{z})=0$. Thus on the boundary $\bar{z}$ can be eliminated and Cauchy's residue theorem can be used even in the case of rotational motion.

For example a circular cylinder of radius $a$ exposed to a stream $U$ on which is superposed uniform shear flow of vorticity $\omega$ undergoes the lift $\pi \rho a^{2} \omega U$.

4. Complex Stokes's theorem. Just as the theorem of Stokes is fundamental in the theory of "solid flow" if I may use that term to contrast with plane flow, so is the form which the theorem assumes in two dimensions in terms of $z$ and $\bar{z}$ a powerful tool. This plane form I have named the complex Stokes's theorem [10]. The complex Stokes's theorem refers to a plane area $S$ bounded by a closed curve $C$ and states that

$$
\int_{C} f(z, \bar{z}) d z=2 i \int_{S} \frac{\partial f}{\partial \bar{z}} d S .
$$

Cauchy's theorem is a particular case, namely when $\partial f / \partial \bar{z}=0$, which 
is the single equation implying the Cauchy-Riemann equations.

One immediate and important application is to the calculation of the kinetic energy [11] of liquid in irrotational motion, with its bearing on virtual mass. Thus

$$
\begin{aligned}
\text { Kinetic energy } & =\frac{1}{2} \rho \int_{S} q^{2} d S=\frac{1}{2} \rho \int_{S} \frac{d w}{d z} \frac{d \bar{w}}{d \bar{z}} d S \\
& =\frac{1}{2} \rho \int_{S} \frac{\partial}{\partial \bar{z}}\left(\bar{w} \frac{d w}{d z}\right) d S=-\frac{1}{4} i \rho \int_{C} \bar{w} d w .
\end{aligned}
$$

If the region $S$ is multiply-connected, suitable barriers must be introduced as part of the boundary $C$. Note that on the boundary $\bar{z}$ is a function of $z$ so that the integral can be evaluated by the residue theorem.

The complex Stokes's theorem effects a simple proof of a theorem on conformal mapping due to Bieberbach [12].

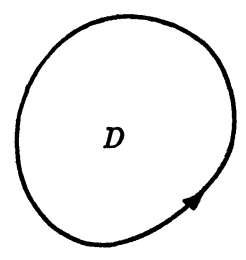

z-plane

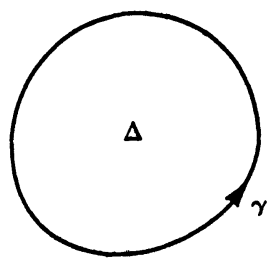

$\zeta$-plane

FIG. 1

Let us map the area $D$ bounded by the contour $C$ in the $z$-plane on the area $\Delta$ bounded by the contour $\gamma$ in the $\zeta$-plane by the relation

$$
z=\zeta+a_{2} \zeta^{2}+a_{3} \zeta^{2}+\cdots=f(\zeta) \text {. }
$$

We then have

$$
2 i D=2 i \int_{D} d S=\int_{C} \bar{z} d z=\int_{\gamma} \bar{f}(\bar{\zeta}) f^{\prime}(\zeta) d \zeta=2 i \int_{\Delta} \bar{f}^{\prime}(\bar{\zeta}) f^{\prime}(\zeta) d S .
$$

Suppose now that $\Delta$ is the circle $|\zeta| \leqq r$. Then on $\gamma, \bar{\zeta}=r^{2} / \zeta$ and therefore by Cauchy's theorem applied to the above integral round $\gamma$

$$
D=\pi r^{2}\left\{1+2 a_{2} \bar{a}_{2} r^{2}+3 a_{3} \bar{a}_{3} r^{4}+\cdots\right\}>\pi r^{2} .
$$

Thus of all areas on which $D$ is mapped by choice of $a_{2}, a_{3}, \cdots$, the circle is the least. Alternatively if we regard $\Delta$ as the given area the 
problem of mapping on a circle $D$ is that of choosing $a_{2}, a_{3}, \cdots$, to make $\int_{\Delta} f^{\prime}(\zeta) \bar{f}^{\prime}(\bar{\zeta}) d S$ a minimum. These are Bieberbach's results.

5. Theorem of Plemelj. Another theorem important for the study of plane flow is due to Plemelj [13]. Let $C$ be a closed contour, $\phi(t)$ a function defined at every point $t$ of $C$, and consider the holomorphic function

$$
\Phi(z)=\frac{1}{2 \pi i} \int_{C} \frac{\phi(t)}{t-z} d t
$$

it being assumed that the integral exists as a Cauchy principal value. Let

$$
\lim _{z \rightarrow t_{0}} \Phi(z)=\Phi^{L}\left(t_{0}\right) \text { or } \Phi^{R}\left(t_{0}\right)
$$

according as $z$ approaches $t_{0}$ from within $C$ or from without i.e. from the left or right. Then Plemelj has proved that (under certain restrictions on $\phi(t))$

$$
\phi\left(t_{0}\right)=\Phi^{L}\left(t_{0}\right)-\Phi^{R}\left(t_{0}\right) .
$$

It easily follows that the function $\Phi(z)$ which satisfies this condition, which is holomorphic in the whole plane (except perhaps on $C$ ), and which vanishes at infinity is given uniquely by $(*)$.

As a simple illustration in potential theory we seek the function $f(z)$ holomorphic within the unit circle $\gamma,|z| \leqq 1$ and zero at infinity, whose real part at the point $t_{0}=e^{i \theta}$ on the circumference is $\sin 2 \theta$. Then

$$
2 \sin 2 \theta=f\left(e^{i \theta}\right)+\bar{f}\left(e^{-i \theta}\right) .
$$

If we continue $f(z)$ outside the circle by the definition

$$
f(z)=-\bar{f}(1 / z),
$$$$
|z|>1
$$

we have

$$
f^{L}\left(t_{0}\right)=\lim _{z \rightarrow t_{0}} f(z),|z|<1 \text { and } f^{R}\left(t_{0}\right)=\lim _{z \rightarrow \infty}-f(1 / z), \quad|z|>1 .
$$

Thus on the circle

$$
f^{L}\left(t_{0}\right)-f^{R}\left(t_{0}\right)=-i\left(t_{0}^{2}-1 / t_{0}^{2}\right)
$$

and so

$$
f(z)=\frac{1}{2 \pi i} \int_{\gamma} \frac{-i\left(t^{2}-1 / t^{2}\right) d t}{t-z}=-i z^{2}
$$


the continuation of which outside the circle is $-i / z^{2}$ which tends to zero as $|z|$ tends to infinity.

Another method for such problems is to apply the formula of Schwarz [14] which is substantially equivalent to the foregoing.

6. Quaternion variable. The generalization of the complex variable to three dimensions leads to Hamilton's quaternions. Alan Rose [15] defines a stream function $(x, y, z, \xi, \eta, \zeta)$ as the flux across the triangle formed by the origin, the point $(x, y, z)$, and the point $(x+\xi, y+\eta$, $z+\zeta)$. If we introduce the vector

$$
\Psi=\left(\psi_{1}, \psi_{2}, \psi_{3}\right)=\left(\frac{\partial \psi}{\partial \xi}, \frac{\partial \psi}{\partial \eta}, \frac{\partial \psi}{\partial \zeta}\right)_{\xi-\eta-\zeta=0},
$$

the velocity is $q=-\nabla \wedge \Psi$.

When the motion is axisymmetrical and irrotational with velocity potential $\phi$, the function

$$
F=\phi+i \psi_{1}+j \psi_{2}+k \psi_{3}
$$

satisfies the condition for it to be a right-regular quaternion [16] function of the quaternion variable

$$
w+i x+j y+k z,
$$

where $w$ is an imagined coordinate whose axis is perpendicular to the axes of $x, y, z$. This condition of right regularity is

$$
\square F=0 \text {, where } \square=\frac{\partial}{\partial w}+\nabla .
$$

This condition, which has four scalar components, may be regarded as the analogue of the Cauchy-Riemann equations for a function $f(z)$ which are in fact the two scalar components of $\nabla f=0$.

Thus the theory of analytic quaternion functions is in principle available.

Rose has shown that it is possible to deduce the perturbation of a uniform stream by a sphere (in terms of the quaternion variable), by starting with a needle-shaped body along the axis of the stream and which therefore does not disturb the uniform flow. This method is entirely analogous to that for deducing the perturbation of a uniform stream by a circle in terms of the complex variable, by starting with a needle-shaped body.

7. Virtual mass. To take the simplest case when a body of mass $M$ moves, with uniform speed $V$ in a straight line, in inviscid liquid the total kinetic energy of the system is of the form $(M+H) V^{2} / 2$ and 
the body moves as if the liquid was absent and the mass of the body were increased from its actual mass $M$ to its virtual mass $M+H$. Here $H$ is the added or hydrodynamic mass for this particular motion and is the coefficient of $V^{2} / 2$ in the expression for the kinetic energy of the liquid.

It is only quite recently that a physical interpretation of hydrodynamic mass as an actual mass of the liquid has been given by Sir Charles Darwin [17]. To understand Darwin's interpretation consider the particular case of a circular cylinder which moves (twodimensional motion) along the $x$-axis from minus to plus infinity. Suppose that when the cylinder is at $x=-\infty$ blue dye is used to color the particles of the fluid in a plane perpendicular to the direction of motion. Since the cylinder in its motion displaces a certain volume of liquid the gap left behind must be filled up, and it might appear reasonable to suppose that when the cylinder has attained the position $x=+\infty$, the blue wall of liquid will have retreated a certain distance to the rear of its initial position. Now the paths of the particles are elasticas as indicated in Fig. 2. So that a particle which is at

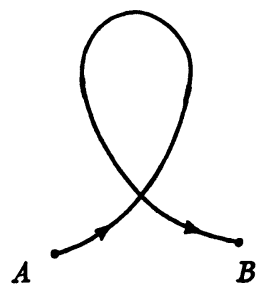

FIG. 2

$A$ when the cylinder is at $x=-\infty$ will have drifted to $B$ when the cylinder is at $x=+\infty$. If then we consider those particles which at a given instant lie in an axial plane of the cylinder perpendicular to the direction of motion, the positions of these particles when the cylinder is at $x=-\infty$ and at $x=+\infty$ define surfaces which, in the plane of the motion are typified by curves $A, A$, and $B, B$ in Fig. 3 .

Thus the particles on the curves $A, A$ move forwards not backwards to the positions $B, B$. The intuitive idea of reflux of a wall of dyed particles is false. Darwin's discovery is this, that the mass of liquid enclosed (per unit thickness of fluid) between the surfaces $A, A$ and $B, B$ is in fact the hydrodynamic mass of the cylinder (per unit thickness) for this particular motion.

The fact can be established by direct integration since the coordinates of the points on the elastica are expressible rationally in 


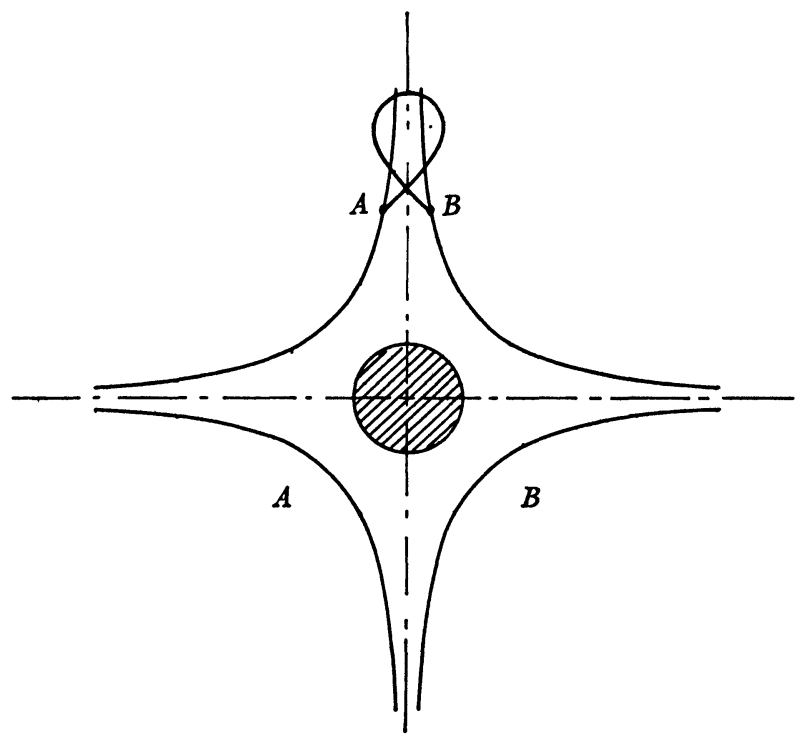

FIG. 3

terms of Jacobian elliptic functions [18]. The argument is however capable of general formulation independent of the particular shape of the cross-section of the cylinder. Moreover as Darwin has shown, the proof can be extended to three-dimensional motion. Thus we have here, I believe for the first time, a genuine physical interpretation of hydrodynamic mass as a mass of fluid entrained by the body.

The corresponding problem of interpretation with a free surface still awaits investigation, although some computations of the hydrodynamic mass itself have been made by Bloh [19] for spheres and ellipsoids half immersed and totally immersed. The problem of rotation also offers opportunities for investigation. If we consider a plate rotating in two-dimensional motion, it is found that two regions $A$ and $B$ (Fig. 4) exist in which the liquid is trapped and moves round

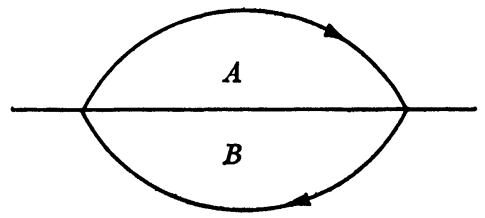

FIG. 4

with the plate, not as a rigid body but consistently with irrotational flow. This points to a method of interpreting hydrodynamic mass due to rotation. 
8. Free streamlines. Problems concerning free streamlines have been intensively studied in recent years in relation to the cavities formed behind bodies moving at high speed and the water entry of missiles.

The plane problem has been greatly simplified by Max Shiffman's method [20] of reflection across free streamlines whereby an image in the free streamline is obtained of the actual flow; in effect a method of analytical continuation of the flow across the free streamline. Apart from the insight-giving character of this method, one of the integrations is already performed by geometrical considerations, thus greatly simplifying the technique. The method also affords a direct geometrical interpretation of the drag coefficient.

To give the simplest possible illustration, it is a provable theorem that the image of flow in an angle is flow outside an equal angle. Thus for a jet running along a wall $A B C$ (Fig. 5) with a free stream-

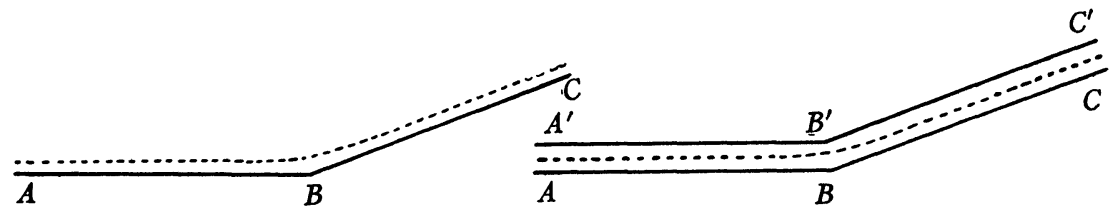

FIG. 5

line (shown dotted) we reduce the problem to flow in a channel formed by the original wall and a parallel wall got by translation along the bisector of the angle $A B C$.

The method also takes care of re-entrant jet problems such as the impact of a stream on a wedge (Fig. 6).

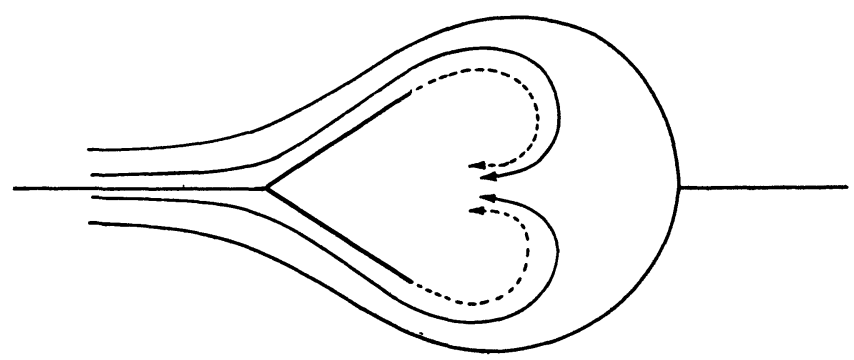

FIG. 6

Applied to the impact of a stream on a lamina the image flow indicates a layer of sources on the down stream face, and thus offers at 


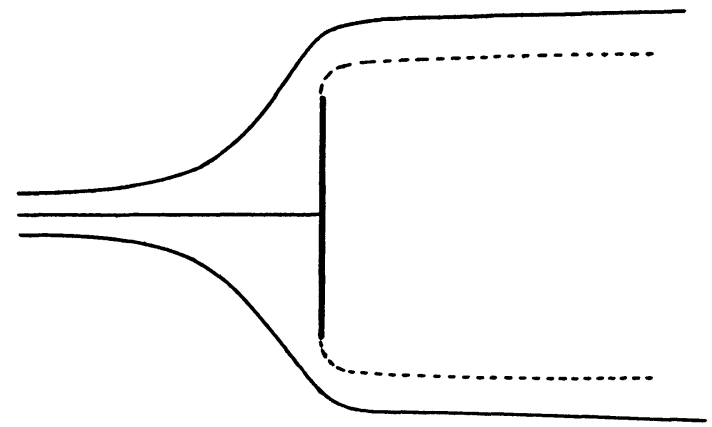

FIG. 7

least a suggestion for tackling the corresponding problem of a stream impinging on a circular disc.

Riabouchinsky [21] 30 years ago discovered a method of finding an approximation to the drag on a lamina exposed to a stream.

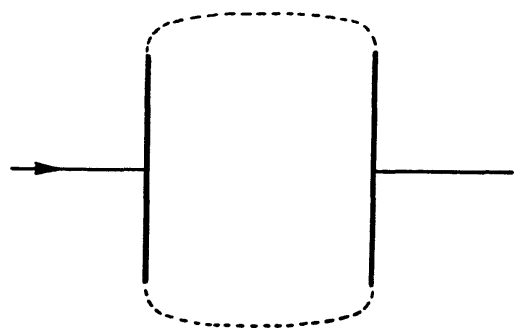

FIG. 8

The method consists in placing an image lamina downstream, the two being joined by free streamlines. This problem is capable of exact solution [22] in terms of Jacobian elliptic functions, where Prandtl's cavitation number $\sigma=\left(p_{\infty}-p_{c}\right) / 2^{-1} \rho V^{2}$, where $p_{\infty}$ is the pressure at infinity and $p_{c}$ is the pressure in the cavity between the free streamlines, appears in the parameter of the elliptic functions. The case of an unlimited cavity can be approximated to any degree of accuracy by increasing the distance between the plate and its image.

Quite recently Garabedian [23] has undertaken the numerical study of the axisymmetrical problem, in particular that of the circular disc with an equal image disc behind it on the Riabouchinsky model. The two discs are joined by a free stream surface to enclose a region containing water vapor. A convergent iterative process is set up, taking an initial form of the free streamline based on the curve afforded by the plane flow solution of Riabouchinsky. 
9. Gravity flow with a free surface. Let us now turn to the problem of free surfaces when the liquid moves under gravity. By a free surface we shall mean a surface which always consists of the same fluid particles and on which the pressure is constant. The grand illustration in nature is the surface of the ocean.

But few simple complete solutions of this problem are known. Two nontrivial cases are Gerstner's trochoidal wave and Rankine's combined vortex. In the Gerstner wave [24] the free surface is a trochoid and the motion is rotational. In Rankine's combined vortex [25] the motion is rotational within a vertical cylindrical core and is irrotational outside the core, reminiscent of Poe's "Maelstrom."

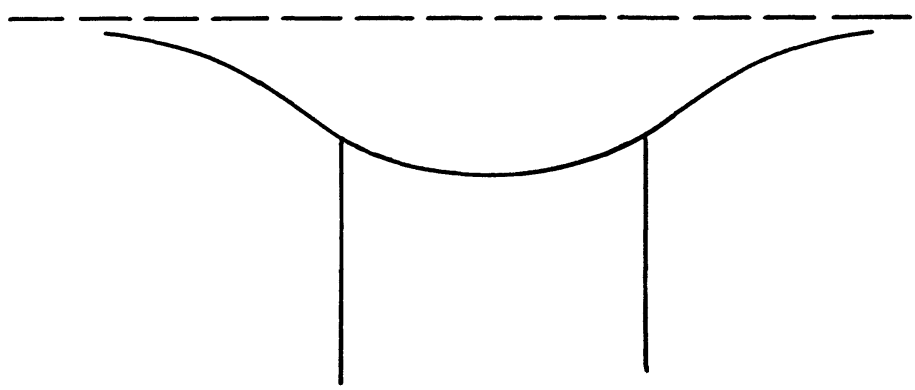

FIG. 9

There are three main problems whose solution still eludes us, namely the problem of periodic surface waves on deep water, surface waves on water of finite depth, and the solitary wave.

Let us examine what is involved in the problem of the two-dimensional progressive wave of permanent type on water of infinite depth. As long ago as 1925 Levi-Civitá [26] stated the boundary value problem in the form

$$
\frac{\partial \theta}{\partial \psi}=-\frac{g}{c^{8}} e^{-8 r} \sin \theta, \quad \psi=0, \quad q=c e^{r} .
$$

Here $\theta$ is the inclination of the velocity vector to the horizontal. By mapping the strip defined by a period on the unit circle and then obtaining a Taylor series expansion Levi-Civitá established the existence of this type of wave.

One might enquire why such an existence theorem should be necessary, when waves are to be seen any day. But it is only fair to remember that the wave here considered is perfectly regular and is propagated in an inviscid fluid, conditions to which observed waves only approximate. 
The intrinsic difficulty of the problem here envisaged is the nonlinearity of the boundary condition, quite apart from the fact that the form of the boundary is part of the solution.

10. Method of T. V. Davies. Write $\omega=\theta+i \tau$. The linearized approximation is obtained by assuming $|\omega|=\left(\theta^{2}+\tau^{2}\right)^{1 / 2}$ to be small of the first order. From this assumption follows the usual theory of waves of small amplitude and slope.

There is, however, a serious limitation to the use of the linearized approximation. A wave will break at the crest when the fluid velocity there exceeds the velocity of advance of the wave. The critical case is when the fluid velocity at the crest is equal to the velocity of the wave that is to say when

$$
q=c e^{\tau}=0
$$

so that $\tau=-\infty$. It follows that no approximation based upon the assumption that $\tau$ is small can throw any light on the case of breaking.

A way to avoid this difficulty has been proposed by T. V. Davies [27], namely in Levi-Civitá's boundary condition to replace $\sin \theta$ by $(\sin 3 \theta) / 3$. This substitution replaces one nonlinear boundary condition by another. It still preserves the essential feature but allows $\tau$ to be large. Mathematically the nonharmonic function $e^{-8 r} \sin \theta$ is replaced by the harmonic function $e^{-8 \tau} \sin 3 \theta$. The boundary condition then becomes

$$
\frac{\partial \theta}{\partial \psi}=-\frac{g}{3 c^{3}} e^{-3 r} \sin 3 \theta, \quad \psi=0,
$$

which leads to

$$
e^{-3 i \omega}=1-3 A \exp (2 \pi i w / c \lambda),
$$

where $w=\phi+i \psi$ is the complex potential, $c^{2}=g \lambda / 2 \pi$, and $\lambda$ is the wave length.

The critical condition for breaking at the crest is $u-i v=0$ when $w=0$, which, since $u-i v=c e^{-i \omega}$, leads to $3 A=1$. In the neighborhood of the crest the wave then forms a wedge of angle $2 \pi / 3$.

Again if we write $A=2 \pi a / \lambda$, where $a$ is small we recover the ordinary linearized theory.

Thus the method discovered by Davies yields an approximation which applies over the whole range from waves of small amplitude to those on the point of breaking at the crest.

The method has also been applied by my colleague B. A. Packham [28] of the Royal Naval College to obtain a unique solution in closed 
form of the problem of the solitary wave on the surface of water of depth $h$, namely

$$
\begin{aligned}
e^{-3 i \omega} & =1-\sin ^{2} k c h \operatorname{sech}^{2} \frac{1}{2} k(w-i c h), \\
c^{2} / g h & =(\tanh k c h) / k c h, \quad 0 \leqq k c h \leqq \pi / 3 .
\end{aligned}
$$

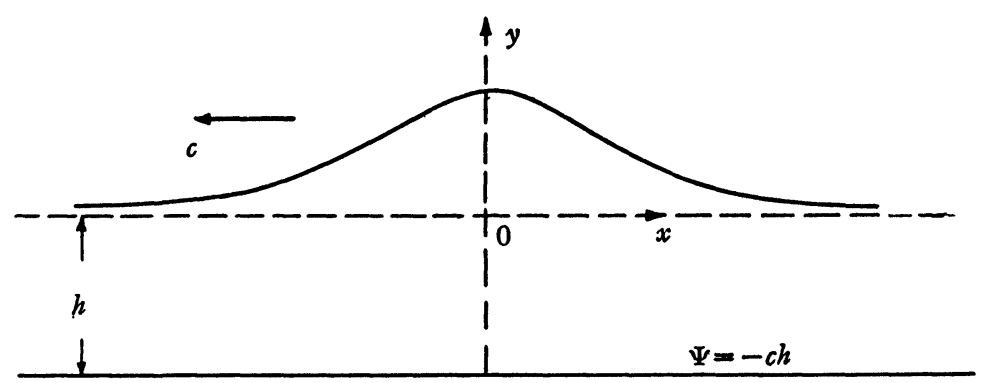

FIG. 10

In this result $k=0$ corresponds to rest and $k c h=\pi / 3$ corresponds to breaking at the crest.

11. Exact methods for free surfaces. We can now turn to exact methods of treating the free surface problem. The equation of motion of an inviscid liquid under gravity can be written in the form [29]

$$
a-\xi=-\frac{1}{\rho} \nabla p,
$$

where $\boldsymbol{a}$ is the acceleration of a fluid particle and $\boldsymbol{g}$ is the gravitational force per unit mass. Since $\nabla p$ is a vector normal to the surfaces of constant pressure, of which the free surface is one, it follows that for particles in the free surface, the vector $a-g$ is normal to the free surface. This remark has been applied by Fritz John [30] to reduce the attack on the two-dimensional case to the solution of a linear partial differential equation of the second order, of parabolic type as follows. If the free surface has the equation

$$
z=f(\alpha, t), \quad z=x+i y,
$$

where $\alpha$ is a real Lagrangian parameter, we have

$$
\frac{\partial^{2} f}{\partial t^{2}}+i g=i r(\alpha, t) \frac{\partial f}{\partial \alpha},
$$


where $r(\alpha, t)$ is an arbitrary real-valued function. Every two-dimensional continuous free surface must satisfy this equation, whether the motion is rotational or irrotational, steady or time-dependent.

When the motion is irrotational it is not difficult to deduce the equation satisfied by the complex potential. John has also shown that the form $y=k(x, t)$ of the free surface can be prescribed. For a particle, $x$ and $y$ are functions of $t$ and the constant pressure condition then implies that $x$, as a function $t$, satisfies a nonlinear ordinary differential equation of the second order.

In the case of irrotational steady motion the problem can be simplified still further to depend on the solution of an ordinary second order linear equation of the form

$$
f^{\prime \prime}(\beta)+i g=i S(\beta) f^{\prime}(\beta),
$$

where $S(\beta)$ is an arbitrary function.

It follows from these considerations that two-dimensional free surface problems can be reduced to the study of a limited class of differential equations. Nevertheless progress will necessarily depend on divining the proper form of the arbitrary function involved.

On the other hand we have here a means of generating an unlimited number of free surfaces by assigning the arbitrary function. In particular, in the steady irrotational case, putting $S(\beta)$ equal to a constant leads to a trochoidal free surface from which we can proceed to a trochoidal progressive wave. Unfortunately this wave has to be associated with a moving ocean bed since the singularities of the progressive wave are no longer fixed.

A different approach to steady irrotational flow is due to H. Lewy [31]. By proper choice of axes and units the surface condition can be expressed in the form

$$
\frac{d z}{d w}\left(\frac{d z}{d w}-2 i \frac{d y}{d w}\right)+\frac{1}{2 y}=0, \quad \psi=0, \quad w=\phi+i \psi .
$$

Let us regard this as an equation in the complex domain involving a complex function $y=-\eta(w)$, real when $\psi=0$. The equation has the solution

$$
z=-i \eta(w)+\int\left[\frac{1}{2 \eta}-\left(\frac{d \eta}{d w}\right)^{2}\right]^{1 / 2} d w .
$$

Provided the integral is real on some segment of $\psi=0$, we have

$$
y=-\eta(w) \text { on } \psi=0
$$

and the point $z$ describes a free streamline. 
Lewy has also proved that the flow is analytic on the free surface in the steady case and has thus established that the formula gives the most general steady irrotational motion with a free surface.

M. J. Vitousek [32] has studied in detail flows obtained by attributing certain forms to $\eta(w)$ and in particular waves of trochoidal and cycloidal profile.

12. Analogies. It sometimes happens that problems in apparently diverse branches of science lead to equations and boundary conditions which have the same mathematical form and differ only in the physical interpretation attributed to the symbols. We then say that an analogy exists. Examples are Kirchhoff's analogy [33] between the equations of equilibrium of a thin rod, straight and prismatic when unstressed, held bent and twisted by forces and couples applied at the ends, and the equations of motion of a heavy rigid body turning about a fixed point: the analogy between the torsion of a prismatic beam [34] and the two-dimensional hydrodynamic problem of a cylinder of the same shape as the surface of the beam, hollow, filled with inviscid liquid, and rotating about an axis parallel to the generators: the analogy between electric circuits [35], electric current, magnetic force, positive magnetic pole, and vortex filaments, strength of vorticity, fluid velocity, source respectively. I quote here as further illustrations two analogies between flow of a viscous and an inviscid liquid.

The first concerns flow in a straight horizontal pipe [36] in which viscous liquid flows steadily under a pressure gradient $P$. The velocity of a particle at distance $r$ from the axis is $\psi-P r^{2} /(4 \mu)$, where $\mu$ is the coefficient of viscosity and $\psi$ is the stream function for inviscid liquid filling the same pipe and rotating about the axis with angular velocity $P /(2)$.

The second analogy concerns the slow motion of a solid of revolution which rotates with angular velocity $\omega$ about its axis of revolution in unbounded viscous liquid [37]. The fluid particles describe circles about the axis of revolution with velocity $\psi / r$, where $r$ is the distance of the particle from the axis and $\psi$ is the stream function when the solid moves in inviscid liquid in the direction of its axis with velocity $-2 \omega$.

13. Motion of a body through a fluid. The classical case, governed by Kirchhoff's equations, when a vessel deeply submerged (e.g. a submarine) moves with velocity $u=u(t)$ and angular velocity $\omega$ in liquid otherwise at rest gives [38] for the force $\boldsymbol{R}_{0}$ and the moment $\boldsymbol{M}_{0}$, exerted by the liquid on the vessel, 


$$
\begin{aligned}
& R_{0}=-\frac{\partial K}{\partial t}-\omega \wedge K, \\
& M_{0}=-\frac{\partial L}{\partial t}-\omega \wedge L-u \wedge K,
\end{aligned}
$$

where

$$
K=\rho \int \phi d S, \quad L=\rho \int \phi r \wedge d S
$$

the integrals being taken over the surface of the body, and $d S$ denoting the vector element of area directed into the fluid. When a surface ship moves with speed less than $(g h)^{1 / 2}$ where $h$ is the depth of the water, it is accompanied by a wave train whose energy is maintained at the expense of the fuel supply of the ship and consequently the ship experiences a wave making resistance. A deeply submerged submarine experiences little resistance from this cause, and that is one reason why the submarine is coming into prominence as a war vessel. M. D. Haskind [39] has this year extended the above formulae to include the case where the liquid has velocity $v=v(t)$ at infinity and consequently acceleration $\boldsymbol{f}=d \boldsymbol{v} / d t$. If $\Phi$ is the velocity potential, he defines $\phi=\Phi-\boldsymbol{v} \cdot \boldsymbol{r}$ and the corresponding force and moment are

$$
\begin{aligned}
R & =R_{0}+M^{\prime} f, \\
M & =M_{0}+v \wedge K+M^{\prime} r \wedge f,
\end{aligned}
$$

where $\boldsymbol{M}_{\mathbf{0}}, \boldsymbol{R}_{\mathbf{0}}$ are calculated from the Kirchhoff formulae, $M^{\prime}$ is the mass of liquid displaced and $\boldsymbol{r}$ is the position vector of the centre of buoyancy.

The motion of a submarine when gravity is taken into account has been discussed also this year by P. V. Harlamov [40]. The problem he studies is that of a body totally immersed with buoyancy equal to the weight of the body so that, in general, the weight and buoyancy form a couple. The results of Harlamov's thorough-going discussion are not suited to reproduction here but I mention one as a specimen. If $\boldsymbol{r}$ is the position vector of the centre of buoyancy relative to the centre of gravity of the body, permanent translation is impossible if the body is immersed so that the plane containing the vector $\boldsymbol{r}$ and one of the principal axes of the virtual mass ellipsoid is vertical but the vector $\boldsymbol{r}$ is not vertical.

14. A general solution of the hydrodynamical equations. Let me conclude this necessarily brief review of some of the methods used in 
hydrodynamics today, (I have purposely kept exclusively to the mathematical aspect), by stating a general result [41].

The equation of motion and the equation of continuity of a fluid, viscous or inviscid, compressible or incompressible, are satisfied identically by

$$
\begin{aligned}
\rho & =\nabla^{2} \chi, \quad \rho q=-\nabla(\partial \chi / \partial t), \\
\Phi & =\rho(q ; q)+\left(V-\partial^{2} \chi / \partial t^{2}\right) I+\nabla \wedge \Psi \wedge \nabla .
\end{aligned}
$$

Here $\Phi$ is the stress tensor, $\chi$ is an arbitrary function, $\Psi$ is an arbitrary 2-tensor, and $V=\epsilon \rho \Omega$, where $\Omega$ is the potential of a conservative field of external force and $\epsilon=1$ if the fluid is incompressible, and $\epsilon=0$ otherwise.

\section{REFERENCES}

1. L. M. Milne-Thomson, Theoretical hydrodynamics, 3d ed., New York, Macmillan, 1956, 19.04. Cited below as M-T, $H$.

2. M-T, $H, 19.76$.

3. L. N. G. Filon, Forces on a cylinder, Proc. Roy. Soc. London Ser. A vol. 113 (1926).

4. $\mathrm{M}-\mathrm{T}, H, 3.44$.

5. M-T, $H, 2.60,2.50$.

6. V. Bjerknes, Vid.-Selsk. Skrifter, Kristiania, 1918.

7. M-T, $H, 5.01$.

8. L. M. Milne-Thomson, Hydrodynamical images, Proc. Cambridge Philos. Soc. vol. 36 (1940); M-T, $H, 6.22$.

9. M-T, $H, 6.41$.

10. M-T, $H$, (1938) 9.51 ; (1956) 5.43 .

11. M-T, $H, 9.10$.

12. L. Bieberbach, Einfilhrung in die konforme Abbildung, (1920).

13. J. Plemelj, Ein Ergänzungssatz zur Cauchyschen Integraldarstellung analytischen Funktionen, Monatshefte für Mathematik und Physik vol. 19 (1908) pp. 205-210.

14. M-T, $H, 11.2$.

15. A. Rose, On the use of a complex (quaternion) velocity potential, Comm. Math. Helv. vol. 24 (1950) pp. 135-147.

16. R. Fueter, Comm. Math. Helv. vol. 7 (1934-1935) p. 307.

17. Charles Darwin, Proc. Cambridge Philos. Soc. vol. 49 (1953) pp. 342-354.

18. M-T, $H, 9.21$.

19. E. L. Bloh, Prik. Mat. Meh. vol. 19 (1955) pp. 353-358.

20. M. Shiffman, Comm. on Applied Math. vol. 1 (1948) pp. 89-99; vol. 2 (1949) pp. 1-11.

21. D. Riabouchinsky, Proc. London Math. Soc. vol. 19 (1921) pp. 206-215.

22. M-T, $H, 12.23$.

23. P. R. Garabedian, Mathematical theory of three-dimensional cavities, and jets, Bull. Amer. Math. Soc. vol. 62 (1956) pp. 219-235.

24. M-T, $H, 14.80$.

25. M-T, $H, 13.13$.

26. T. Levi-Civitá, Math. Ann. vol. 93 (1925) p. 264.

27. T. V. Davies, Proc. Roy. Soc. London Ser. A vol. 208 (1951) p. 475. 
28. B. A. Packham, Proc. Roy. Soc. London Ser. A vol. 213 (1952) p. 238.

29. M-T, $H, 14.82$ and footnote on p. 412.

30. F. John, Two-dimensional potential flows with a free boundary, Comm. Pure App. Math. vol. 6 (1953) pp. 497-503.

31. H. Lewy, Comm. Pure App. Math. vol. 5 (1952) p. 413.

32. M. J. Vitousek, Some flows in a gravity field satisfying the exact free surface condition, Stanford University, 1954.

33. A. E. H. Love, Mathematical theory of elasticity, 4th ed., Cambridge, 1934, pp. $399-408$.

34. Ibid. p. 314.

35. $\mathrm{M}-\mathrm{T}, H, 18.24$.

36. $\mathrm{M}-\mathrm{T}, H, 19.32$.

37. W. D. Collins, On the steady rotation of a sphere in a viscous fluid, Mathematika vol. 2 (1955) pp. 42-47.

38. M-T, $H, 17.43$.

39. M. D. Haskind, Neustanovivకeesya dviženie tverdogo tela v uskorennom potoke bezgranicnoi zidkosti, Prik. Mat. Meh. vol. 20 (1956) pp. 120-123.

40. P. V. Harlamov, Postypatel'nye dviženiya tyaželogo tverdogo tela v żidkosti, Prik. Mat. Meh. vol. 20 (1956) pp. 124-129.

41. L. M. Milne-Thomson, Journal of Fluid Mechanics vol. 2 (1957) p. 88.

BROWN UNIVERSITY 